\title{
Development of Disease Self-Management (DSM) Regulatory, Non-communicable Diseases Negotiable Emerging eHealth driven Food Processing Technologies
}

\author{
Amol Dagadkhair ${ }^{*}$, Shradha Rodge ${ }^{1}$, and Vasant Pawar ${ }^{1}$ \\ ${ }^{1}$ MIT School of Food Technology, MIT Art, Design and Technology University, Pune, MH, India- 412201
}

\begin{abstract}
In the modern food technology digital era, innovation and consumerism driven hypothesis generation plays important role to make the system summarily applicable under information and communication technologies (ICT) domain. eHealth conceptualization and promulgation based on scientific and technological information dissemination, recorded as a need base intervention to update food technology scenario. Nutrition transition leading to goodness and smartness has direct relationship with health of the consumer. Moreover, lifestyle transition generative non-communicable diseases (NCD's) is becoming a vibrant challenge to food technologists. The sedentary status and individual inactivity jointly invited physionutritional health problems like NCD's world over. Disease self-management (DSM) by eDiet requires hand in hand association of innovative food technology products and processes. eHealth appears to be one stop solution to prevent, cure and stimulate non-recurrence through regulatory dietary pattern. This may also be justify more efficiently with regulatory mechanism of DSM. Food processing being an art of material transition, leading to consumable security, requires to bring it under health claim base food processing scenario. Hence, concerted efforts of eHealth and novel food processing technologies are rightly awaiting to modify the DSM to ensure health security to consumers. The review base focus on tailoring of eHealth and novel food processing technologies is a techno-economical option to develop a regulatory mechanism for negotiation of NCD's in future generation.
\end{abstract}

\section{Introduction}

The complete well-being of not only physical and mental state but also social status and not just a free from diseases or infirmity is nothing but the health (WHO). The fulfillment in the most imperative reachable standard of living is one of the significant benefits of every person without race, religion, and political opinion, budgetary or social state of a person. Unequal development in various nations in the advancement of health and control of infections, particularly communicable diseases, is a typical peril. Governments have a commitment with respect to the quality of their society which can be fulfilled interestingly by the plan of acceptable well-being and social measures [19].

eHealth is a process of better utilization of Information and Communication Technologies (ICT) for the sake of health. It practices in alliance with worldwide, regional and national agencies to promote and strengthen the usage of ICT in health improvement. This unit is situated at the Department of Service Delivery and Safety in the Cluster of Health Systems and Innovation [20]. In the World Health Assembly, 2018, it has been recorded that the digital technologies are playing an important role in improving society health. The present delegates also have agreed on development and implementation of digital technologies to improve status of NCD's. [7].

The dreadful Noncommunicable diseases (NCDs), includes coronary heart diseases, cancer, stroke, diabetes and different lung diseases, which are reason behind death of about $70 \%$ of all deaths around the world. In the case, $75 \%$ of whole NCD's deaths, and $82 \%$ of the 16 million individuals who died early, or before accomplishing the age 70 years, in specially low and middle income nations. The rising of NCDs has been driven by on a very basic level four noteworthy peril factors viz. constant use of tobacco, physical inactivity, over consumption of alcohol and deplorable weight control plans [21]. Disease Self-management is a practice which includes dynamic, collaborative, and regular process in which people deals to cope up a chronic diseases. Self-management refers to "the capability of the person, to interact with family, society, and healthcare professionals, to control symptoms, treatments, lifestyle changes, and psychosocial, cultural, and spiritual consequences of health conditions" [3]. Thus present review paper enlightens the various eHeath technologies, DSM and advanced food processing technologies can bring a possible solution on NCD's. 


\section{Emerging eHealth Technologies}

Advanced innovations are always developing and finding new applications in health care services, even while the industries are battling with selection and 'digital transformation'. Every year new applications rise, yet the fundamental advancements driving them continue as before. In 2019, they surveyed many organizations around the globe with single question: "Please indicate the key technology which you believe will have the most profound impact on the healthcare industry during 2019". [14].

\section{1 mHealth}

mHealth is a one of eHealth fragment. Till today, nobody has defined mHealth. For the survey purpose, the Global Observatory for eHealth (GOe) has characterized mHealth or mobile health as public and medical wellbeing practice upheld by mobiles, personal digital assistants (PDAs), patient monitoring devices and different remote gadgets. It also includes utilization of SMS and voice services with 3G, 4G, Global Positioning System (GPS), Bluetooth technology and General Packet Radio Service (GPRS) [9].

The text messages on mobiles is one of common approach for DSM, either with or without any other suitable technology. Current researches on T2DM and T1DM reported that the text messages and demonstration are having an impressive elevation in diet and exercise, an increase in weekly exercise and introduction of fruits and vegetables in regular diet, helps in minimizing fat intake and regulating blood sugar level. Also, there was a continuous improvement in the desired positive behaviors. Additionally, with the quantity of days members practiced every week expanding from two days to very nearly four days, and the sugar level of members who consumed products of fruits and vegetables daily expanding ranged from $61 \%$ to $79 \%$. The scores for diabetes self-care increased for healthy diet taking and practicing 30 minute exercise regularly. [2,10,18].

The European Heart Network (EHN) [4] has noted that an effective implementation of e/mHealth solutions could increase the prevention and remedies of CVD's. Mobile applications approached by people of different age groups, together with educated or health conscious individuals. Moreover, smartphone applications may help out the CVD's patients to understand the do's and don't after a cardiac attack, and they could find possible solution to it immediately.

\subsection{Websites}

Websites are the information delivering online systems through various media including audiovisual and written content. Moreover advancement in technology allows the web-based programs access by mobile devices. It has been observed that number of internet-based technologies are being used for the transfer of DSM instruction. Most of the studies are concentrated on change in glycemic level than in dietary pattern and physical activity.
Furthermore it is recorded that educating people through internet based technologies found more efficient than typical care, with the capacity to attain additional patients with more convenience and efficacy [8].

\subsection{Wearable Technologies}

Wearable innovations involves pedometers, accelerometers, and shrewd watches are creating as novel ways to deal with assistance DSM by self-regulating physical movement and diet and outfitting the customer with brief updates, reminder and input. [11] An analysis of randomized selected eleven controlled trials associated with pedometer use, physical activity and glycemic control in person with T2DM. A consistent pedometer use resulted in an increased physical activity (increased by 1,800 steps/ day). Fascinatingly, it shows that it affected physical activity than without objective setting. Notwithstanding this ascent in physical activity, there was little or no change in HbA1c content, despite the fact that subgroup with objective setting remembered for their intervention.

\subsection{Virtual Reality}

The virtual reality is a computerized formation of the total virtual condition which recreates reality (Audio-visual) without sharing the entirety of its physical characteristics. In certain occurrences, the client can explore through and cooperate with a virtual condition. T2DM disease selfmanagement training could be conveyed in an augmented experience condition or in a traditional in-person position. The study was conducted in association with multidisciplinary group (dietitian, dialectologist, and medical attendant) to convey care to the member in an underlying individual counsel, trailed by eight groups per week for several weeks. The eHealth mediation utilized food displays with exercise amenities in the virtual sphere. A face-to-face sessions were organized for the control group in a gathering passage. Furthermore it was observed that physical activity was lengthened by $18 \%$ in virtual, though it diminished by $25 \%$ with respect the face-to-face communication; in any case, there were not any contrasts between dietary plans [15].

\subsection{Virtual Restaurants and Food Nutrition}

The virtual restaurants like Ubereats, Zomato, Food Panda, Swiggy etc. should depict the information about foods they are processing on their webpage/application while purchasing the food item. The said initiative will make easy approach for consumers to purchase the required foods. India is country with young population (more than $50 \%$ by 24 years and above) and increasing urbanization with intense speed [1]. The same things are responsible for increase in demand for convenience foods such as virtual restaurant. The virtual restaurants have to come up with an initiative to incorporate nutritional information about products on their websites/ applications to segregate the products by consumer as per their requirement. Hence the food items obtained through these 
virtual restaurants are examples of eDiet which will serve the consumers with their prime motto.

\subsection{Short Term Online e-courses}

The short term online courses are readily approachable on e-platforms like Coursera, Swayam, FSSAI video library etc. regarding food safety and quality (health claims) awareness. The courses are helping the consumers to know more about their disease, treatment, and norecurrence. FSSAI's video Library is available to everyone with objective to create a sustainable culture and habit of eating safe and healthy foods by endorsing individual awareness as well as collective action. This won't just empower customer to settle on informed choices but also toughen the institutional system to promote, spread and support safe food and healthy diets [5].

\subsection{Digital posters/panels}

Displaying the digital posters/ panel regarding food quality and safety at eating places like Restaurants, Hotels, Mess, Public gatherings (Railway platform, Bus Depot, Airports etc.) to increases awareness about different processed foods. Example, one of novel initiative carried by FSSAI namely Eat Right India has to be spread through such digital posters/panels.

\section{Novel food processing}

\subsection{Impact of Conventional Food Processing Technologies on Food, Nutrition and Health}

Food processing is an art performed to covert raw foods into consumable foods from thousands of years. Foods processing not only makes foods ready to consume but also changes its physical, chemical and microbiological characteristics is such a way that it drastically affects its nutritional content and makes foods nutrient deficient. Most of water soluble nutrients (vitamins and minerals) are getting destroyed in thermal processing [12].

\subsection{Novel Drying Techniques}

The novel food drying techniques includes microwave, vacuum, freeze, ultrasound assisted drying, heat pump drying, high intensity electric field drying, and refractance window drying are making food processors more capable to minimize the nutrition losses while processing.

\subsection{Nano-technology}

The quick improvement in nanotechnology has been encouraging the changes of traditional food areas, especially smart packaging, active packaging, nanosensors,. Various new nanomaterials have been created to improve food food quality and safety. Moreover, it has recently studied that nanotechnology has made its approach towards pathogen detection, food packaging, disease treatments and bioactive compounds transfer to target sites [7].

\subsection{Cold Plasma Technology}

Initially cold plasma was utilized for cleansing the delicate things and now it is stretched out to food processing sector as an emerging technology. For a considerable length of time it has been seen as helpful for microbial inactivation while keeping up natural qualities of new products. In this method cold gases are used purify the surfaces of packaging or food products items. The procedure can possibly inactivate microorganism without raising temperature above $40^{\circ} \mathrm{C}[13]$.

\subsection{Artificial Intelligence and Robotics in Food Processing}

AI is an advanced computational technique has wide applications in different manufacturing industries including food processing industries. In food processing it has numerous application from sorting and grading, cleaning, manufacturing, quality analysis, GC, HPLC, to food safety and quality evaluation of foods and packaging materials too [17].

Innovative technology in various areas have extend their application skyline of robotics to an incredible extent. Robots basically have the ability to transmute the food processing, handling, etc. practices. Therefore, robotics is emerging technology in deployment of robots in food sector. Subsequently, the features belonging to robots are kinematics, hygiene, dynamics, human-robot interaction, economic efficiency, operation and maintenance and safety and protection are of great significance. Now a day's food processing segment having potential where numerous research opportunities are open from various technology perspective. It is new potential territory where plentiful research openings in 'robo-food' by coordinating headways from different innovation spaces [6].

\section{Conclusion}

The intention behind this review article was to exemplify the various benefits and opportunities in the e-health technology domain and novel food processing technologies. An amalgamation of these two emerging technologies can satisfy the need of individual who is eclipsed by the different NCD's.

\section{References}

1. Anonymous, Report on Indian Food Services Industry: Engine for Economic Growth \& Employment: A Roadmap for Unlocking Growth Opportunities, FICCI, New Delhi, India (2019).

2. E. Burner, M. Menchine, E. Taylor and A. Arora, J Diabet Sci Technol. 7(1), 111-118. (2013)

3. S. G. Dena, J. Sarah, J. N. Scholarsh. 44(2), 136-144 (2012). 
4. European Heart Network. Cardiovascular patients and eHealth - a European Heart Network paper. Available at: http://www.ehnheart.org/ component/downloads/downloads/1438 (2013).

5. FSSAI Video Library, https://fssai.gov.in/ fssaivideolibrary/about (2019).

6. I. Jamshed, H. Zeashan, K. Azfar, Food Sci Technol. 37(2), 159-165 (2016).

7. L. Rashidi, K. Darani. Crit Rev Food Sci Nutr. 51, 723-730 (2011).

8. E. R. Megan, J. A. Elroy, L. W. Rebecca, W. Katie, K. Michelle, C. Robin, E. C. Clare. Diabet Metab Synd Ob, 9, 381-390 (2016).

9. mHealth, WHO New horizons for health through mobile technologies, Global Observatory for eHealth series, World Health Organization, 3, 6-7 (2019).

10. S. Nundy, A. Mishra, P. Hogan, S. Lee, M. Solomon, M. Peek, Diabet Edu. 40(6), 806-819 ( 2014)

11. S. Qiu, X. Cai, X. Chen, B. Yang, Z. Sun, BMC Med. 12, 36 (2014).

12. R. Devi, Sch J Agric Vet Sci. 2(4A), 304-311 (2015).

13. R. Thirumdas, C. Sarangapani, U. Annapure, Food Biophy, 10, 1-11 (2014).
14. R. Das. https:// www.forbes.com/sites/ reenitadas/ 2019/02/04/ the-top-five- digital- healthtechnologies- in- 2019/\#24af9aab6c0f (2019).

15. M. Rosal, R. Heyden, R. Mejilla, M. Rizzo, C. Veerappa, M. Wiecha. JMIR Res Protoc. 1(2), e24. (2012).

16. Seventy one World Health Assembly, https://www.who.int/about/governance/worldhealth-assembly/seventy-first (2018).

17. A. Sofu, D. Gida, S. Cilt. Applications of Artificial Intelligence in Food Science and Technology Area (Turkish with English Abstract). agris.fao.org. (2007).

18. K. Waki, H. Fujita, Y. Uchimura, K. Omae, E. Aramaki, S. Kato, H. Lee, T. Kobayashi, K. Ohe, J Diabet Sci Technol. 8(2), 209-215 ( 2014)

19. WHO https://www.who.int/ about/ who-we-are/ constitution (2019a).

20. WHO https://www.who.int/ehealth/en/ (2019b).

21. WHO https://www.who.int/ncds/en/ (2019c). 